\title{
Dielectric Barrier Discharge in Atmospheric Air for Different Barrier Materials
}

\author{
V.Yu. Khomich, M.V. Malashin, S.I. Moshrunov*, E.A. Shershunova \\ Institute for Electrophysics and Electric Power of Russian Academy of Sciences, \\ St.-Petersburg, Dvortsovaya nab., 18, 191186 Russia
}

\begin{abstract}
This paper deals with the ignition of a diffuse dielectric barrier discharge in air at atmospheric pressure with a high voltage rectangular pulse supply. The study is focused on the effects of dielectric materials. The current and voltage waveforms of discharges with different barriers look similar. But the discharge development process can be different. It is described by many synchronous electron avalanches in a case of alumina ceramics, and by a streamer - in a case of acrylic glass.
\end{abstract}

DOI: 10.12693 /APhysPolA.127.1298

PACS: 52.80.Tn

\section{Introduction}

Dielectric barrier discharge (DBD) has been widely used for pumping of gas lasers, in surface treatment and in plasma displays $[1,2]$. Besides, the DBD is a promising tool in plasma medicine, plasma chemistry and in aerodynamics as an ion source for the creation of control gas dynamic flows [3, 4].

In our work we already reported about producing the high current diffuse volume DBD in air at atmospheric pressure, room temperature under conditions of natural humidity in a simple plane-parallel electrode arrangement, using a high voltage solid-state supply, generating rectangular microsecond pulses with nanosecond rise and fall time [5]. This paper describes the further investigation of the discharge. The shape of the applied pulses allowed estimate a delay of the discharge appearance. Besides, the barrier material effect on the DBD mode was investigated.

\section{Experimental setup}

The experimental setup is similar to the used in our previous work [5]. A rectangular voltage pulses are applied to the upper electrode through series resistor $R$ with a preset value (Fig. 1) and the ground electrode [5-7].

The DBD arrangement comprises two identical planeparallel aluminum electrodes with the diameter of $30 \mathrm{~mm}$. The lower (ground) electrode is covered with a $2 \mathrm{~mm}$ dielectric plate of $50 \mathrm{~mm}$ widths. The distance $h$ between the dielectric surface and the upper electrode is equal to $1 \mathrm{~mm}$. The experiments were conducted in air at atmospheric pressure under natural humidity of 40-60 percent for two barrier materials: $\mathrm{Al}_{2} \mathrm{O}_{3}$ ceramics and acrylic glass. The value of effective air gap capacitance $C_{a}$ (Fig. 1) for both materials is $6 \mathrm{pF}$. Effective barrier capacitance $C_{b}$ equals to $27 \mathrm{pF}$ and $11 \mathrm{pF}$ for ceramics and acrylic glass correspondingly.

*corresponding author; e-mail: serg-moshkunov@yandex.ru
The input voltage hereinafter referred to as $V_{i n}$. Its value means the storage capacitor voltage commutated by switch to $R$ and electrode arrangement.

The discharge current was measured by low inductance current sensor. The voltage applied to the discharge gap (DG) was recorded by the high voltage probe (Tektronics P6015A). The signals from voltage probe and current sensor were observed by LeCroy WaveRunner 104Xi-A oscilloscope (bandwidth of $1 \mathrm{GHz}$, sampling rate of $10 \mathrm{GS} / \mathrm{s}$ ).

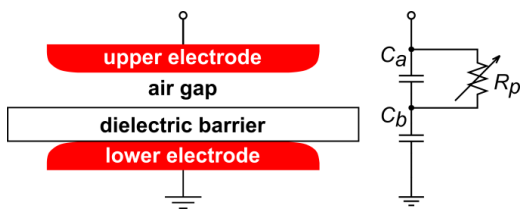

Fig. 1. Electrode arrangement and its equivalent electric scheme.

\section{Results and discussion}

The diffuse volume DBD was obtained for both types of dielectrics at pulse repetition rates of tens of hertz. However, the similar results were obtained also in a singlepulse mode with preliminary removal of the charge from the dielectric surface. The last is needed to insure the absence of a residual charge at the dielectric barrier.

The DBD mode in air was found to depend strongly on $R$ value. The volume diffuse DBD was observed at relatively low $R$ values, whereas filamentary mode with incomplete filling of the DG by the plasma - for high $R$ values. So the diffuse mode was realized at $R=80-500 \mathrm{Ohm}$. Its typical waveforms are presented in Fig. 2a and Fig. 3a. At $R$ about $1 \mathrm{kOhm}$ the discharge current trace begins to disintegrate into separate pulses, and bright channels start to appear in the background of residual diffuse glow. With further increase of the resistance up to $10 \mathrm{kOhm}$ and higher the DBD becomes filamentary. At $R=13 \mathrm{kOhm}$ the plasma filaments in the DG are visible with the naked eye $(2 \mathrm{~b}, 3 \mathrm{~b})$. The current signal, observed during the experiment, consists of two components. The first one, being in temporal coincidence with voltage rise at DG, corresponds to charging the capacity of DG (equals to connected in series $C_{a}$ and 
$C_{b}$ ). And the second component - to the gas discharge itself.

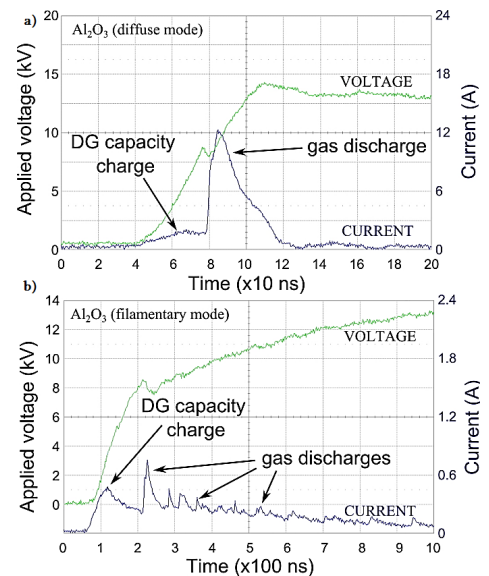

Fig. 2. Waveforms of DBD in air with $\mathrm{Al}_{2} \mathrm{O}_{3}$ as a dielectric barrier: a - diffuse mode $\left(V_{i n}=12 \mathrm{kV}\right.$, $R=80 \mathrm{Ohm}), \mathrm{b}$ - filamentary mode $\left(V_{i n}=14 \mathrm{kV}\right.$, $R=13 \mathrm{kOhm})$.

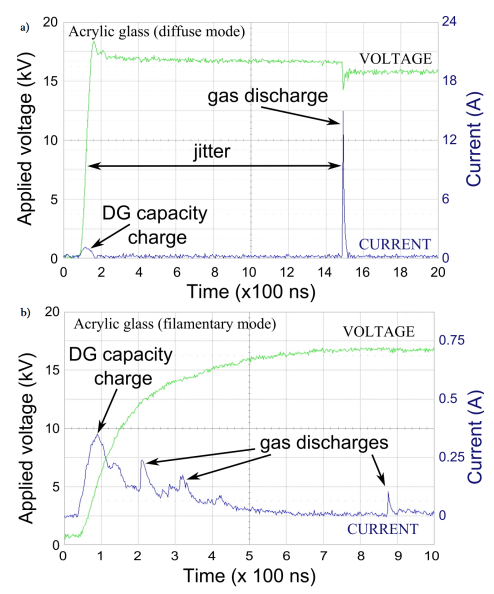

Fig. 3. Waveforms of DBD in air with acrylic glass as a dielectric barrier: a - diffuse mode $\left(V_{i n}=16 \mathrm{kV}\right.$, $R=150 \mathrm{Ohm}), \mathrm{b}$ - filamentary mode $\left(V_{\text {in }}=16 \mathrm{kV}\right.$, $R=13 \mathrm{kOhm})$.

Delays of the discharge current were found to depend on the experiment conditions. For $\mathrm{Al}_{2} \mathrm{O}_{3}$ the start of the discharge processes occurred at the rising edge of the voltage pulse at the $\mathrm{DG}\left(V_{i n}=12 \mathrm{kV}-16 \mathrm{kV}\right)$ or just after it $\left(V_{i n}=6-8 \mathrm{kV}\right)$. For acrylic glass the discharge occurs with much bigger time delays after beginning of the voltage pulse and has some jitter (Fig. 3a).

In the case of the diffuse DBD, the current, flowing through the discharge gap, looks like a solitary bellshaped pulse with amplitude of $13 \mathrm{~A}$ and FWHM of about $20 \mathrm{~ns}$ for $\mathrm{Al}_{2} \mathrm{O}_{3}$ (Fig. 2a) and $15 \mathrm{~A}$ and $15 \mathrm{~ns}$ for acrylic glass (Fig. 3a) correspondingly. In the case of the filamentary DBD, the current signal looks like many single randomly delayed pulses (Fig. 2b, Fig. 3b).

Experimental values of charge $Q$, transferred by the discharge process, and the peak discharge current as a function of input voltage $V_{i n}$ and $R$ are shown in Fig. 4a and Fig. $4 \mathrm{~b}$.

The value of charge $Q$ corresponds to the charge transferred in one discharge pulse in the case of the diffuse mode and to the summary charge of several most significant discharge current pulses in filamentary mode. For the filamentary mode the maximum current amplitude, observed during the applied voltage pulse, was considered as the peak current value.

With increase of $R$ the peak value of the discharge current for acrylic glass does not change significantly, whereas the charge transferred during the discharge process decreases. This is caused by reducing the duration of the discharge current pulse.

The calculated curves of the maximum charge that can be carried by the DBD, are also presented in Fig. $2 \mathrm{a}$ and Fig. 2b. These dependencies were obtained according to the simple assumption that the voltage across the electrodes dropped to zero at the end of the discharge pulse. Two extreme cases were considered: 1. When the discharge started just at the beginning of the voltage rise, the limit charge equaled to:

$$
Q_{\lim 1}=C_{b} V_{i n} \text {. }
$$

2. When the discharge took place after the voltage growth, the limit charge calculation resulted in:

$$
\begin{aligned}
& Q_{\lim 2}=C_{b}\left(V_{i n}-V_{b}\right)= \\
& C_{b} V_{i n}-\frac{C_{a} C_{b}}{C_{a}+C_{b}} V_{i n}=\frac{C_{b}^{2} V_{i n}}{C_{a}+C_{b}},
\end{aligned}
$$

where $\frac{C_{a} C_{b}}{C_{a}+C_{b}} V_{i n}$ - the charge, transferred during charging the equivalent capacitance of the DBD $\left(\mathrm{C}_{a}\right.$ and $\mathrm{C}_{b}$ in series) under no discharge condition and $V_{b}$ - voltage at $\mathrm{C}_{b}$ after charging the equivalent capacitance.

In comparison with the first case, current pulses corresponding to charging the equivalent capacitance of the DBD and the discharge itself are clearly separated. This fact allows deducting from the total transferred charge the component necessary for charging the equivalent circuit. So, the limit charge in the second case is less.

The difference between the experimental value of the transferred charge and the limit charge is caused by the residual electric field in the gap, insufficient for maintaining further discharge processes, and also by the presence of resistor $R$ in the external circuit.

As can be seen from Fig. 4 for the same barrier material and at the same $V_{i n}$, the electric charge $Q$, carried during the discharge process, decreases with increasing of $R$.

In the diffuse mode capacity $C_{b}$ is charged to a voltage close to $V_{i n}$, and the whole dielectric surface is being charged. This causes reducing of the electric field strength in the discharge gap and abruption of the discharge processes despite the voltage applied to the electrodes. As a result, there are no provision for repeating discharges.

In cases of substantial limitation of avalanche processes (large values of $R$ ), the charge, transferred for a single discharge pulse, is not able to provide a compensation field across the whole volume of DG. Therefore, with 
reducing the current of the single pulse the breakdown electric field strength in the DG is retained at least in a general part of the gap volume, which leads to initiation of new electronic avalanches and corresponding pulses of the discharge current trace. Thus, for large $R$ the typical

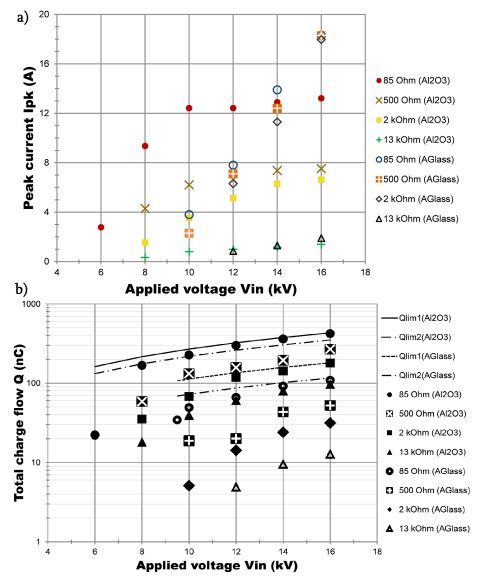

Fig. 4. Calculated and experimental dependences of charge transferred during the discharge (a) and the peak discharge current (b) on voltage $V_{i n}$.

discharge current waveform consists of multiple pulses with much less amplitudes as compared to the case of low $R$. In this case, as we can see from Fig. 4, the charge value carried during the discharge is much lower that the limit one. So, the residual voltage in the DG is slightly changed by the discharge pulses, what makes possible the initiation of next discharges.

According to the experimental data (characteristic times and rates of the discharge processes) the most likely pattern of the discharge process looks like multiplication of avalanches in the whole volume of the air gap up to the moment corresponding the peak current in the waveform.

Considering the discharge formation mechanism the following correlations have been used [1]:

$$
\begin{aligned}
& \mathrm{e}^{\alpha h_{c r}}=N_{c r}, \\
& \alpha=15 p \exp \left(-\frac{365}{E / p}\right) .
\end{aligned}
$$

Where $\alpha$ - ionization coefficient, $N_{c r}$ - number of electrons needed for avalanche-to-streamer transformation, $E$ - electric field strength, $p$ - ambient pressure, $h_{c r}-$ critical value of the air gap.

When $h<h_{c r}$ the mechanism of discharge development - an electron avalanche, whereas under $h>h_{c r}-$ streamer one. The Eq. 4 is an empirical formula for calculation of the ionization coefficient under atmospheric air conditions.

Substituting the values $N_{c r} \approx 10^{8}, E=7 \times 10^{4} \mathrm{kV} / \mathrm{cm}$ (typical prebreakdown field strength in the air gap in the case of alumina oxide as a barrier, evaluated from equivalent scheme Fig. 1) and $p=760$ Torr into Eq. 3 and 4 we get $\alpha=218 \mathrm{~cm}^{-1}, h_{c r} \approx 1 \mathrm{~mm}$.

The same evaluation for acrylic glass with $E=9 \times 10^{4} \mathrm{kV} / \mathrm{cm}$ (typical prebreakdown field strength in the air gap in the case of acrylic glass as a barrier) gives $\alpha=470 \mathrm{~cm}^{-1}$ and $h_{c r} \approx 0.4 \mathrm{~mm}$.

The evaluation described above does not take into consideration electron quenching in electronegative gases like air, so effective $h_{c r}$ will be upward than values which have been obtained.

Hence, according to the simplified criteria (3), (4) the mechanism of the discharge formation for alumina oxide is an avalanche multiplication. For acrylic glass barrier the main mechanism of the discharge propagation is a streamer one. However, the discharge current traces for both dielectrics are quite familiar, except for the large and random delays in the case of acrylic glass barrier. So, these statements require experimental verification.

The defining role in the development of the discharge plays conditions in the DG if plasma resistance $R_{p} \gg R$ (see Fig. 1) or in the external circuit if $R_{p} \ll R$.

\section{Conclusion}

Thus, the volume diffuse DBD for two types of dielectric material (alumina ceramic and acrylic glass) was achieved and investigated. The parameters of the external circuit were shown to influence strongly on the DBD mode. The DBD was realized in two regimes: at repetition rates of tens of hertz and at a single-pulse supply mode with preliminary removal of the charge from the dielectric surface.

Noteworthy that discharge ignition occurs at the leading edge of the voltage pulse for ceramics whereas some delay takes place for acrylic glass.

Our evaluation of the barrier discharge formation mechanism in air shows that it looks like many synchronous electron avalanches for alumina oxide whereas for acrylic glass the streamer takes place. These statements require experimental verification, because the shape of the discharge current traces and the external appearance of glow for both dielectric materials are very similar.

\section{Acknowledgments}

This work was supported by the Russian Foundation for Basic Research, grant No. 13-08-01043.

\section{References}

[1] Yu.P. Raizer, in: Gas Discharge Physics 3rd ed. Intellect, Dolgoprudny, Russia, 2009.

[2] U. Kogelschatz, B. Eliasson, W. Egli, Pure Appl. Chem. 71, 1819 (1999).

[3] S.I. Moshkunov, S.V. Nebogatkin, I.E. Rebrov, V.Yu. Khomich, V.A. Yamshchikov, Plasma Physics Reports 38, 1040 (2012).

[4] S.I. Moshkunov, S.V. Nebogatkin, I.E. Rebrov, V.Yu. Khomich, V.A. Yamshchikov, Quantum Electronics 41, 1093 (2011).

[5] M.V. Malashin, S.I. Moshkunov, V.Yu. Khomich, E.A. Shershunova, V.A. Yamshchikov, Technical Physics Letters 39, 252 (2013).

[6] E.V. Ivanov, S.I. Moshkunov, V.Yu. Khomich, Instruments and Experimental Technics 49, 88 (2006).

[7] S.I. Moshkunov, V. Yu Khomich, V.A. Yamshchikov, Quantum Electronics 41, 366 (2011). 